\title{
Seclusion Room in Psychiatric Setting (National Center for Mental Health): Policy Analysis
}

\author{
Anas H. Khalifeh ${ }^{1,2^{*}}$, Malek M. Khalil ${ }^{2,3}$ \\ ${ }^{1}$ Department of Nursing, Prince Hamzah Hospital, Ministry of Health, Amman, Jordan \\ ${ }^{2}$ School of Nursing, The University of Jordan, Amman, Jordan \\ ${ }^{3}$ Faculty of Nursing, Zarqa University, Zarqa, Jordan \\ Email: *anaskhalifeh@yahoo.com
}

How to cite this paper: Khalifeh, A.H. and Khalil, M.M. (2018) Seclusion Room in Psychiatric Setting (National Center for Mental Health): Policy Analysis. Open Journal of Nursing, 8, 697-707. https://doi.org/10.4236/ojn.2018.89052

\section{Received: July 26, 2018}

Accepted: September 26, 2018

Published: September 29, 2018

Copyright (c) 2018 by authors and Scientific Research Publishing Inc. This work is licensed under the Creative Commons Attribution International License (CC BY 4.0).

http://creativecommons.org/licenses/by/4.0/

\begin{abstract}
Background: Improving the quality of care in psychiatric settings is the most important goal of policy through the delivery of a comprehensive care, treatment, control, protection, and rehabilitation of patients with mental disorders. The main concern in mental health care is the continuing use of seclusion and the slow pace of change. Purpose: Analyze the seclusion room policy in National Center for Mental Health (NCMH) in Jordan to recognize the issue and present alternative solutions in order to modify and improve the current seclusion room policy. Method: The authors got seclusion room policy from $\mathrm{NCMH}$; the method in this analysis will be used six-step model; and then searched the database for alternatives using EBSCO, PUBMED, MEDLINE, CINAHL, and Ovid. Result: The authors discuss use medications, training program, manipulate environment, de-escalation technique, and status quo alternatives that helps in minimizing use of seclusion, decreasing the incidence of aggressive behaviors occurrences, and decreasing unsafe behaviors against health care providers in the psychiatric settings. Conclusion: Manipulates environment is the best alternative after evaluated alternatives according to criteria.
\end{abstract}

\section{Keywords}

Seclusion, Policy, Analysis, Psychiatric, Psychiatric Settings

\section{Introduction}

Today health policy makers face overwhelming reality to attain decisions in a very short time; therefore; there is a need for policy analysis to highlight on important policy issues, enhance the implementation process of policy, and lead to 
better health outcomes [1]. Also, the cope with main concerns of access, quality, and cost are most important claims for health policy analysts which are support innovative ideas [2]. According to World Health Organization (WHO), health policy is defined as the set of main objectives, plans, and activities that are assumed to achieve specific goals of a quality health care within a government or society [3]. Moreover, policy analysis is identified as the process and attempt to identify, obtain, and evaluate alternative policies or programs to determine which alternative policies will achieve goals that are proposed to determine the most effective, efficient, and feasible alternative [4]. In the last decade improving the quality of care in psychiatric settings is the most important goal of policy through the delivery of a comprehensive care, treatment, control, protection, and rehabilitation of patients with mental disorders [3].

The prevalence of mental disorders increases in worldwide [5]; most common of these disorders are schizophrenia, mood, and anxiety disorders [6]. In Jordan, the most commonly assigned diagnosed at hospitals admission are schizophrenia and related disorders (49\%) and mood disorders (35\%) [7]. Hence, an important issue in psychiatric inpatient settings is aggressive events among patients with severe mental disorders [8]. However, controlling and managing this behavior use several interventions including seclusion, physical restraints, chemical restraints, and time out [9]. Seclusion is a widespread measure used in psychiatric settings.

\section{Purpose}

The purpose of this paper is to analyze the seclusion room policy in National Center for Mental Health (NCMH) in Jordan to recognize the issue and present alternative solutions in order to modify and improve the current seclusion room policy.

\section{Method}

The authors got seclusion room policy from NCMH [10], the method in this analysis will be used six-step model that developed by Patton et al. 2015, and then searched the database for alternatives using EBSCO, PUBMED, MEDLINE, CINAHL, and Ovid. The six steps of policy analysis are: verify, define and detail the problem; establish evaluation criteria, identify alternative policies, assess alternative policies, display and distinguish among alternatives, and implement, monitor, and evaluate the policy as presented in Figure 1.

\section{Setting}

National Center for Mental Health (NCMH) located in Al-Balqa governorate, Jordan, contain 239 beds and 167 nurses working in this hospital, involve 7 departments, 4 for male and 3 for female, the cases distribute between acute and chronic [11]. A number of admissions in 2011 around 1807 compared with 2010 was 1622 [11]. In addition, approximately $42 \%$ of patients spend less than one year, $1 \%$ of patients spend 1 - 4 years, $1 \%$ of patients spend $5-10$ years, and $56 \%$ 


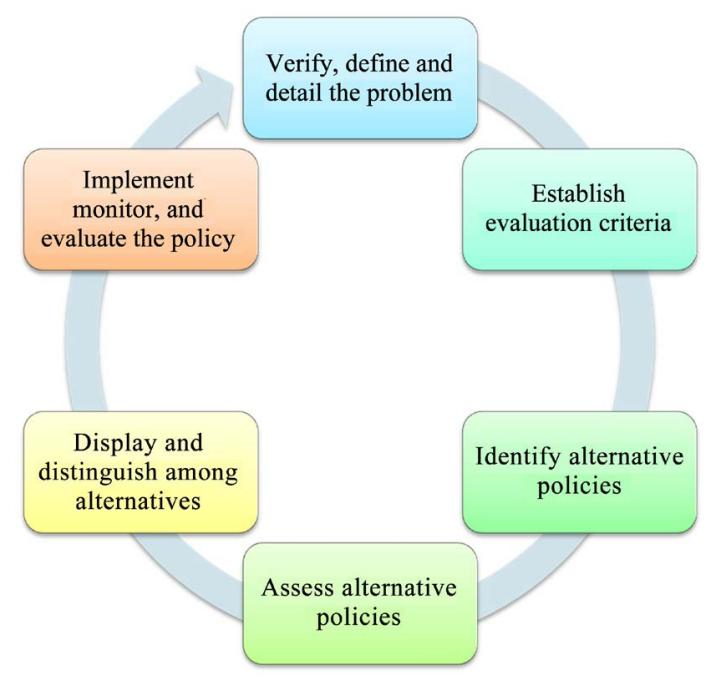

Figure 1. Steps of Policy Analysis (Patton et al., 2015).

of patients spend more than 10 years in the hospital [11], in each department there is a seclusion room.

\section{Results}

The authors analyze policy according to steps and found articles and policies about seclusion room. The implementation and evaluation of alternative approaches to the care of patients are necessary to reduce seclusion and introduce changes to practice.

\subsection{Verify, Define and Detail the Problem}

Aggressive events considered one of the most challenging issues for healthcare team in psychiatric inpatient units [12]. Aggression is defined as a forceful and complex behavior or attitude that is physically or verbally toward oneself or against others [13]. The interventions to control of aggressive associated with an increased incidence of injury to both patient and health care providers who deal with them [14]. Its aim is to help patients, and it is a lawful and legitimate technique used in mental health services.

Seclusion of the most useful intervention is defined as isolating a patient away from other patients in locked rooms which are specially prepared and separated from other patients [15]. The indications of seclusion use are to prevent harm to self and others, to prevent damage the physical environment, to prevent a serious disruption of the treatment program, as an emergency in the dangerous behaviors, to decrease of stimulation, and the patient's request [16]. The priority in mental health hospitals is safety, and there is no effective treatment without a safety [17]. However, this intervention is controversial, although the health care providers is intended to protect patients and other patients; they restrict freedom and are usually applied against a patient's will. This considered a serious humanitarian, ethical, and legal issue for patients, their caregivers, clinicians, and po- 
licymakers [18] [19].

The main concern in mental health care is the continuing use of seclusion and the slow pace of change [20]. In Netherland, about $27 \%$ of patients in psychiatric units have experience with seclusion [21]. Moreover, in 2012 approximately 51\% of psychiatric hospitals in Germany used seclusion [22]. In Jordan, $6 \%-10 \%$ of patients in psychiatric hospitals in the Ministry of Health hospitals were physically restrained or secluded [7]. Khudhur reported that the majority of Jordanian psychiatric nurses worked in mental health services used seclusion for reducing environmental stimuli and reduce anxiety associated with other persons; also, they considered seclusion very important intervention. Moreover, the causes for using the seclusion are violence and destruction [23]. On the other hand, fifty-nine percent of Dutch psychiatric clinicians prefer seclusion as the method of containment, so the Dutch government to improve the quality of care supported to prevent seclusion [24].

Policy change involves the restriction or regulation of seclusion prompts the development of seclusion reduction initiatives. Also, the mean conflict will show up between all the health care providers and stakeholders. The current policy has negative outcomes and does not cover all conditions. Given these undesirable negative outcomes, new ideas will establish to resolve negative outcomes. The authors will add some modifications to the current policy and suggest possible alternatives and additional choices for the policy in a clear way.

\subsection{Establish Evaluation Criteria}

The authors in this part compare, measure, and select among alternatives and also consider administrative ease, cost and benefits, effectiveness, equity, legality, and political acceptability. Furthermore, recognize desirable and undesirable outcomes. The desirable outcomes for the current policy are increasing the best response and the safe way to the patient and to the health team, provide a protocol or checklist clearly stated to use it when some become unsafe to him and to the others during hospitalization and decrease the chance of damage to the physical environment. About the undesirable outcome may be the inability of the health team to perform the seclusion protocol, criteria or checklist accurately which may give a confused evaluation outcome about the patient and the uneducated health care providers about the seclusion cause may the inappropriate dealing with these cases can cause harm to both health care providers and the patient and increase the incidence of seclusion.

Evaluation of the seclusion room policy in $\mathrm{NCMH}$, for administrative ease the current policy does not require time and skills, each department has a specific room to perform the seclusion. About cost and benefits criteria, applying the objectives of this policy it's not cost and to obtaining more benefits does not need spending more money. The effectiveness of policy is presented by applying objective of policy that achieves the desired outcomes. About equity, the policy is providing safety for the patient, nurses, psychiatrists, other patients, and other 
caregivers in NCMH but may cause harm. The legality of current policy is confirmed as any policies in NCMH because the policy it is certified by Jordanian nursing council and Jordanian ministry of health. Finally, there is no conflict with any political collision to applying this policy.

\subsection{Identify Alternative Policies}

The authors discuss some solutions and strategies of seclusion room policy and to generate better solutions and modification on current policy that helps in minimizing use of seclusion, decrease the incidence of aggressive behaviors occurrences, and decrease unsafe behaviors against health care providers in the psychiatric settings. The authors will list some alternative strategies in this section and evaluate these alternatives in next section. The following alternatives are based on policies utilizing in other hospitals and evidence-based practice.

Use medications: This alternative is an effective way to suppress aggressive behavior, sometimes used in emergency situations like agitation but not for the purpose of restrain [19]. Most common medications that used are: benzodiazepine, typical antipsychotic drugs like haloperidol and a typical antipsychotic can be used like clonazepam, quetiapine, and risperidone.

Training program: Novice nurses and any expert nurse once come to work in psychiatric settings he/she see himself a novice because it is a new area of practice, so those nurses and all health care providers working there need a training program to know how to adapt to aggressive behaviors, how to build therapeutic relationship and communication which are effective in reducing the aggressive behaviors in psychiatric patients and may be used seclusion only as a last resort when all other intervention attempts have been made. In addition, train the health care providers clinical supervision strategy that helps in managing expected crisis [25].

Manipulate environment: Assess and keep the environment free from any stimuli which induce calming and decrease impulses for the patient. Furthermore, try to keep the clinical settings calm with less coercive and control rules because studies confirmed that areas with more coercive and control rules are more likely to stimulate patients for aggressive behaviors that may cause health care providers injuries [25].

De-escalation technique: This technique is a strategy used by the health care providers to calm an angry patient or a way to prevent highly charged or highly aroused person to commit a dangerous act by supporting through talking, listening, and using a supportive way. There are some steps of the verbal de-escalation technique: assess the patient situation immediately and intervene by using a calm voice, use problem-solving technique, be empathetic with patient, calm and reassure the patient that no harm or hurt will come to him/her, and offering help to the patient and do not challenge and use argumentative stances [26].

Status quo: If the previous alternatives are failed and do not workable, it is important to use intervention which has used in NCMH. The current seclusion 
policy stated that the seclusion room should be locked after last health care providers leaves the room, and it is trying to use the least restrictive method to help the patient, such as removing the patient from a disturbing environment, having the patient spend time in room, providing close supervision, or getting the patient involved in some activity with supervision. Usually, that attitude mostly aggravates patient to commit aggression behavior due to feeling that he or she in a prison. However, minor modification to the status quo could be implemented [10].

\subsection{Assess Alternative Policies}

Assessment of all alternatives strategies of seclusion room policy and estimate expected outcomes of each policy alternative. Also, it is necessary to evaluate how each possible alternative benefit the criteria previously established. The assessment for each alternative will be done by using the same measures of administrative ease, cost and benefits, effectiveness, equity, legality, and political acceptability. In this section summarizes the evaluation of the alternatives in Table 1 and summarizes the expected outcomes for the alternatives in Table 2.

Table 1. Evaluation of alternatives.

\begin{tabular}{|c|c|c|c|c|c|c|}
\hline $\begin{array}{c}\text { Criteria } \\
\text { Alternatives }\end{array}$ & Administrative ease & Cost effectiveness & Effectiveness & Equity & Legality & $\begin{array}{c}\text { Political } \\
\text { acceptability }\end{array}$ \\
\hline Use Medications & Easy & $\begin{array}{c}\text { Dependent on the } \\
\text { drug type }\end{array}$ & Effective & Not equal & Legal & Acceptable \\
\hline Training Program & Easy & Expensive & Effective & Yes & Legal & Acceptable \\
\hline $\begin{array}{l}\text { Manipulate } \\
\text { Environment }\end{array}$ & Easy & Cost effective & Partially effective & Yes & Legal & Acceptable \\
\hline $\begin{array}{c}\text { De-Escalation } \\
\text { Technique }\end{array}$ & Easy & Expensive & Effective & Yes & Legal & Acceptable \\
\hline Status Quo & Easy & Cost effective & Partially effective & Not equal & Legal & Acceptable \\
\hline
\end{tabular}

Table 2. Expected outcomes.

\begin{tabular}{|c|c|c|}
\hline $\begin{array}{l}\text { Outcomes } \\
\text { Alternatives }\end{array}$ & Desirable Outcomes & Undesirable Outcomes \\
\hline Use Medications & Reduce use of seclusion and safe & $\begin{array}{l}\text { Inappropriate use this alternative by health } \\
\text { care providers to reduce tasks and efforts }\end{array}$ \\
\hline Training Program & $\begin{array}{l}\text { Decrease injury for patients and } \\
\text { health care providers }\end{array}$ & $\begin{array}{l}\text { Does not use it as trained and } \\
\text { use inappropriate way }\end{array}$ \\
\hline Manipulate Environment & $\begin{array}{l}\text { Calming and reducing the behaviors } \\
\text { that effect on patient oneself and others, } \\
\text { and reduce recourse to seclusion }\end{array}$ & $\begin{array}{l}\text { May is not appropriate for all patients, may effect } \\
\text { on patients' health, and increase incidence of injury } \\
\text { to both patients and health care providers }\end{array}$ \\
\hline De-Escalation Technique & Improve safety in practice & $\begin{array}{l}\text { Does not use effectively and not be able } \\
\text { to entirely eliminate the use of seclusion }\end{array}$ \\
\hline Status Quo & $\begin{array}{c}\text { Safe way to patient and health team providers, } \\
\text { decrease the chance of damage to the } \\
\text { physical environment }\end{array}$ & $\begin{array}{l}\text { Inability to perform the seclusion protocol, } \\
\text { inappropriate dealing with cases, and increase } \\
\text { the incidence of seclusion }\end{array}$ \\
\hline
\end{tabular}


Use medications: Administrative ease, according to health care providers it is simple and does not need extra administrative skills to administer these medications by different routes like intramuscular (IM) and intravenous (IV) to the aggressive patient. Cost effectiveness, using the medication in psychiatric settings is must and does not cost a lot of money but is cost more than current policy. Effectiveness, it is safe for patients and health care providers. Equity, this alternative is safe for both aggressive patients but does not guarantee the safety of the health care providers and do not inflict harm for patients. Legality, it is legal to use some medications to suppress aggressive behaviors, the medications used are safe to the patients and all hospitals attached to the Jordan ministry of health are using these medications. Political acceptability, there is no conflict with the politicians in administering medications.

Training program: Administrative ease, it is effective and easy to apply, it sets the health care providers in a real situation through a role play and training sessions, it is more valuable than traditional lectures and make the health care providers more aware previously and expect many reactions from patients that will encourage the health care providers for applying many techniques that minimize the inflict of aggressiveness. Cost effectiveness, the training programs for health care providers consumed a lot of money and may consider financial burden for the ministry of health. Effectiveness, this alternative is effective to reduce the use of seclusion and prevent aggressive behaviors by teaching health care providers how to interact and communicate with patients through role play activities. It is a safe technique for both health care providers and patients. Equity, the alternative provides and increases skills for health care provider to increase the safety for both health care providers and aggressive patients. Legality, it is legal, useful and safe for both health care providers and patients. Political acceptability, it is not in a conflict with politicians in applying training strategy.

Manipulate environment: Administrative ease, decrease the number of patients in patient rooms, remove anything stimulate the patient to be aggressive like high TV voice and decrease number of students in patient rooms, does not need skills and it is easy to apply. Cost effectiveness, according to this alternative does not need a lot of money and save the budget of the ministry of health. Effectiveness reduce the presence of any stimuli in the area surrounding the patients, it is an alternative for minimizing the occurrence of aggressive behaviors. Equity, when manipulating in an environment decreases aggressiveness which is safe for both health care providers. Legality, it is legal, it is applied in all hospitals that attached to Jordan ministry of health and useful and safe. Political acceptability, it is not to contradict with politicians in applying environment manipulation.

De-escalation technique: Administrative ease, it is easy to apply by health care providers after taking training courses and role play sessions about how to apply de-escalation technique. Cost effectiveness, applying of de-escalation technique it is cost than current policy in training but then it is safe money and budget of the ministry of health. Effectiveness, using verbal de-escalation tech- 
nique for health care providers in psychiatric settings is effective to reduce using seclusion and prevent aggressive behaviors. Equity, the applying of this alternative does not inflict any harm, so it is considered fair for both health care providers and patients. Legality, this alternative can be applied by any health care providers trained or took the course, the course applied by continuous education settings in all hospitals that attached to the ministry of health, so it is legal, useful, and safe. Political acceptability, the applying of this technique does not contradict with politicians.

Status quo: Kept using the current policy with minor modifications, this use if alternatives fail to apply. The minor modification was taken from other policies from Jordan applied in King Abdullah University Hospital and Jordan University Hospital and comparable with evidence based. Both hospitals involved psychiatric settings; the patients are admitted complain from different disorders with different severity. The modifications are seclusion as open-door seclusion with one to one observation in quite room, possible contraindications for locked room seclusion, debriefing and debriefing occurs as soon as possible, no longer than 24 hours after seclusion. In addition, apply current policy if get higher score compared with other alternatives.

\subsection{Display and Distinguish among Alternatives}

Evaluation of alternatives mentioned in last two sections, in this section, compare and summarizes alternatives that help in distinguishing among several alternatives. In addition, strengths and weaknesses will discuss of each alternative as presented in Table 3. So, comparing the alternatives will lead to determining the appropriate one.

The first alternative of use medications has a low score for implementation, because of the weakness points of cost and usage and loses the trust relationship between the patient and the health care providers. Furthermore, in long term may affect physically on the patient and does not achieve the desired outcomes which still the problem not solve [19]. The second alternative training program has a high score of implementations compared with other alternatives but has a problem related to cost that may hamper applicability [25].

Table 3. Strength and weakness of alternatives.

$\left.\begin{array}{cccc}\hline \text { Alternatives } & \text { Strength } & \text { Weakness } & \text { Evidence } \\ \hline \text { Use Medications } & \text { Applicable } & \begin{array}{c}\text { Expensive according to medication use and health } \\ \text { care providers exploits it for personal benefit }\end{array} & {[19]} \\ \text { Training Program } & \begin{array}{c}\text { Enhance knowledge and skills, } \\ \text { sustainability of resources } \\ \text { Applicable and accepted for all } \\ \text { health care providers } \\ \text { Manipulate Environment }\end{array} & \text { Turnover of trained health care providers } & \text { Not accepted for all patients } \\ \text { De-Escalation Technique } & \begin{array}{c}\text { Manage to reduce of seclusion } \\ \text { usage and safe } \\ \text { Save effort and time to } \\ \text { establish new policy }\end{array} & \text { Not appropriate for the budget } & {[25]} \\ \text { Status Quo } & \text { The problem still } & {[26]}\end{array}\right]$


The third alternative manipulates environment cover the weakness of the previous alternative but may not appropriate for all cases [25]. The fourth alternative has weakness look like training program alternative which not covers previous weakness [26]. The last alternative is status quo compared with other alternatives has the lowest score of implication [10]. Finally, the best decision is training program, manipulates the environment, and de-escalation technique these alternatives have the highest score, but training program and de-escalation technique are not appropriate for the budget. Finally, manipulates environment is the best alternative.

\subsection{Implement, Monitor, and Evaluate the Policy}

The approval will get from the ministry of health committee to implement the modified seclusion room policy in NCMH. At the beginning the authors will meet the health care providers to start our move in the policy change, resolve the conflict point, identify the major changes and modifications to the policy, educate the health care providers about the policy, include chart, protocol, and how to implement it at accurate therapeutic way, identify who is responsible for performing it and how to apply the alternative on efficient way to maximize our care and to decrease the seclusion as we can.

Then the authors will draw a monitoring system to have a direct observation of the policy changes, every shift, reviewed every 24 hours from the nurse manager, then reviewed weekly from the executive manager and the policy maker team how and who has the maximum benefit to apply it, the patient, health team providers, and both, does it effectively or not.

\section{Conclusions}

This paper analyzed the seclusion room policy in NCMH in Jordan and recognized the issue and presented alternative solutions to modify and improve the current seclusion room policy. The analysis process used six-step model (Patton et al., 2015), and searched for alternatives using the database EBSCO, PUBMED, MEDLINE, CINAHL, and Ovid. Some solutions and strategies discussed and generated better solutions and modification on current policy that helps in minimizing use of seclusion, decreasing the incidence of aggressive behaviors occurrences, and decreasing unsafe behaviors against health care providers in the psychiatric settings.

The authors listed alternative and evaluated these alternatives. The alternatives were based on policies utilizing in other hospitals and evidence-based practice. The alternatives use medications, training program, manipulate environment, de-Escalation technique, and status quo. Each alternative evaluated according to criteria of administrative ease, cost and benefits, effectiveness, equity, legality, and political acceptability. Manipulates environment is the best alterna-

tive; however, training program and de-escalation technique these alternatives have the high score but were not appropriate for the budget. 
Implementation the modified seclusion room policy in NCMH requires approval from the ministry of health committee. The authors will educate the health care providers about the policy and how to implement it at an accurate therapeutic way, and identify who is responsible for performing it and how to apply the alternative on efficient way to maximize our care and to decrease the seclusion as we can. The authors will draw a monitoring system to have a direct observation of the policy changes.

\section{Conflicts of Interest}

The authors declare no conflicts of interest regarding the publication of this paper.

\section{References}

[1] Collins, T. (2005) Health Policy Analysis: A Simple Tool for Policy Makers. Public Health, 119, 192-196. https://doi.org/10.1016/j.puhe.2004.03.006

[2] Columbia University (2017) Health Policy Analysis, Columbia University Mailman School of Public Health.

https://www.mailman.columbia.edu/become-student/degrees/masters-programs/m asters-public-health/columbia-mph/certificates/health-1

[3] WHO (2005) Mental Health Policy, Plans and Programmes. 2nd Edition, World Health Organization, Geneva, 18-122.

http://www.who.int/mental_health/policy/services/essentialpackage1v1/en/

[4] Patton, C., Sawicki, D. and Clark, J. (2015) Basic Methods of Policy Analysis and Planning. Routledge, London.

[5] Alonso, J. (2012) Burden of Mental Disorders Based on the World Mental Health Surveys. Revista Brasileira de Psiquiatria, 34, 7-11. https://doi.org/10.1590/S1516-44462012000100003

[6] National Alliance on Mental Illness (NAMI) (2017) Mental Health by the Numbers. http://www.nami.org/Learn-More/Mental-Health-By-the-Numbers

[7] WHO (2011) WHO-AIMS Report on Mental Health System in Jordan. World Health Organization, Geneva.

[8] Amoo, G. and Fatoye, F.O. (2010) Aggressive Behaviour and Mental Illness: A Study of In-Patients at Aro Neuropsychiatric Hospital, Abeokuta. Nigerian journal of Clinical Practice, 13, 351-355.

[9] Migon, M., Coutinho, E., Huf, G., Adams, C., Cunha, G. and Allen, M. (2008) Factors Associated with the Use of Physical Restraints for Agitated Patients in Psychiatric Emergency Rooms. General Hospital Psychiatry, 30, 263-268.

https://doi.org/10.1016/j.genhosppsych.2007.12.005

[10] National Center for Mental Health (NCMH) (2011) Policies and Procedures for Psychiatric Nursing. Jordanian Nursing Council, Amman, 1-165.

[11] $\mathrm{MOH}$ (2011) Ministry of Health. http://apps.moh.gov.jo/MOH/En/publications.php

[12] Al-Sagarat, A., Hamdan-Mansour, A., Al-Sarayreh, F., Nawafleh, H. and Moxham, L. (2015) Prevalence of Aggressive Behaviours among Inpatients with Psychiatric Disorders: A Case Study Analysis from Jordan. Nursing \& Health Sciences, 18, 172-179. https://doi.org/10.1111/nhs.12239

[13] Belete, H., Mulat, H., Fanta, T., Yimer, S., Shimelash, T., Ali, T. and Tewabe, T. 
(2016) Magnitude and Associated Factors of Aggressive Behaviour among Patients with Bipolar Disorder at Amanual Mental Specialized Hospital, Outpatient Department, Addis Ababa, Ethiopia: Cross-Sectional Study. BMC Psychiatry, 16, 443. https://doi.org/10.1186/s12888-016-1151-8

[14] Knox, D. and Holloman, G. (2012) Use and Avoidance of Seclusion and Restraint: Consensus Statement of the American Association for Emergency Psychiatry Project BETA Seclusion and Restraint Workgroup. Western Journal of Emergency Medicine, 13, 35-40. https://doi.org/10.5811/westjem.2011.9.6867

[15] Bowers, L., Van Der Merwe, M., Nijman, H., Hamilton, B., Noorthorn, E., Stewart, D. and Muir-Cochrane, E. (2010) The Practice of Seclusion and Time-Out on English Acute Psychiatric Wards: The City-128 Study. Archives of Psychiatric Nursing, 24, 275-286. https://doi.org/10.1016/j.apnu.2009.09.003

[16] American Psychiatric Association (APA) (2006) The Use of Restraint and Seclusion in Correctional Mental Health Care. American Psychiatric Association, Washington DC.

http://www.psychiatry.org/File\%20Library/Learn/Archives/rd2006_Seclusion.pdf

[17] Khalifeh, A. (2015) Position Statement: The Use of Seclusion in Psychiatric Settings. Middle East Journal of Nursing, 9, 31-36. https://doi.org/10.5742/MEJN.2015.92662

[18] Happell, B. and Harrow, A. (2010) Nurses' Attitudes to the Use of Seclusion: A Review of the Literature. International Journal of Mental Health Nursing, 19, 162-168. https://doi.org/10.1111/j.1447-0349.2010.00669.x

[19] Georgieva, I., Mulder, C.L. and Noorthoorn, E. (2013) Reducing Seclusion through Involuntary Medication: A Randomized Clinical Trial. Psychiatry Research, 205, 48-53. https://doi.org/10.1016/j.psychres.2012.08.002

[20] Vruwink, F., Mulder, C., Noorthoorn, E., Uitenbroek, D. and Nijman, H. (2012) The Effects of a Nationwide Program to Reduce Seclusion in the Netherlands. BMC Psychiatry, 12, 231. https://doi.org/10.1186/1471-244X-12-231

[21] Janssen, W., Noorthoorn, E., de Vries, W., Hutschemeakers, G., Lendemeijer, H. and Widdershoven, G. (2008) The Use of Seclusion in the Netherlands Compared to Countries in and outside Europe. International Journal of Law and Psychiatry, 31, 463-470. https://doi.org/10.1016/j.ijlp.2008.09.002

[22] Steinert, T. and Schmid, P. (2014) Coercive Measures in Psychiatric Clinics in Germany: Current Practice. Der Nervenarzt, 85, 621-629. https://doi.org/10.1007/s00115-013-3867-8

[23] Khudhur, I. (2013) Nurses' Knowledge about Psychiatric Patient Seclusion in Jordan. Kufa Journal for Nursing Sciences, 3, 109-113.

[24] Voskes, Y., Kemper, M., Landeweer, E. and Widdershoven, G. (2013) Preventing Seclusion in Psychiatry. Nursing Ethics, 21, 766-773. https://doi.org/10.1177/0969733013493217

[25] Goulet, M., Larue, C. and Dumais, A. (2017) Evaluation of Seclusion and Restraint Reduction Programs in Mental Health: A Systematic Review. Aggression and Violent Behavior. https://doi.org/10.1016/j.avb.2017.01.019

[26] Spencer, S. and Johnson, P. (2016) De-Escalation Techniques for Managing Aggression. Cochrane Database of Systematic Reviews, No. 1, CD012034. https://doi.org/10.1002/14651858.CD012034 